\title{
Editorial
}

\section{Are Artemisinin-Based Combination Therapies For Malaria Beginning To Fail in Africa?}

\author{
Philip J. Rosenthal* \\ Department of Medicine, University of California, San Francisco, California
}

\begin{abstract}
Despite advances in many countries, notably the recent declaration that China is now malaria free, malaria remains entrenched in Africa, where over $90 \%$ of malaria morbidity and mortality is seen. ${ }^{1}$ In the past, Africa suffered greatly from the effects of resistance to chloroquine, the mainstay of malaria treatment for decades, with increasing mortality from Plasmodium falciparum over the last decades of the 20th century. These increases were turned around early this century, with an estimated $57 \%$ decrease in the rate of malaria deaths from 2000 to $2015 .^{2}$ A major factor contributing to this decrease was availability of highly effective artemisinin-based combination therapies (ACTs) to treat malaria.
\end{abstract}

Although ACTs helped to stem the rising tide of malaria mortality in Africa, they faltered in Asia, with delayed clearance of parasites after treatment with artemisinins, generally referred to as artemisinin resistance (or partial resistance), first identified in Cambodia more than a decade ago. Important studies have documented the spread of artemisinin resistance through the Greater Mekong subregion, ${ }^{3}$ the association of resistance with mutations in the $P$. falciparum kelch13 (K13) gene, ${ }^{4}$ and loss of treatment efficacy of some ACTs, notably artesunate-mefloquine ${ }^{5}$ and dihydroartemisinin-piperaquine, ${ }^{6}$ as emergence of resistance to partner drugs followed that to artemisinins.

Of great concern is the potential for spread of ACT resistance (failures due to resistance to both components of the combination) to Africa, with a repeat of what was seen with chloroquine resistance in the last century, with many excess deaths due to the inability of available therapies to effectively treat falciparum malaria. At present, most evidence suggests that multiple ACTs continue to offer excellent therapeutic efficacy against malaria in Africa. ${ }^{7,8}$ Yet, some recent reports suggest that we may be seeing early signs of ACT resistance, as will be discussed below.

More than 100 different mutations have been identified in the $P$. falciparum $\mathrm{K} 13$ gene, with 10 deemed validated markers of artemisinin resistance and another 11, with more limited available data, considered candidate or associated markers by the WHO. ${ }^{8}$ Recently, some of these mutations have been seen to emerge in Africa. The $\mathrm{K} 13561 \mathrm{H}$ mutation, a validated resistance marker, has been seen at up to $20 \%$ prevalence at sites in Rwanda, and it was associated with delayed parasite clearance (but not treatment failure of artemether-lumefantrine) in a clinical trial. ${ }^{9,10}$ Two other $\mathrm{K} 13$ mutations that are candidate (675V) or associated (469Y) resistance markers have been seen at prevalences over $10 \%$ at multiple sites in northern Uganda, ${ }^{11}$ and unpublished reports have described additional relevant mutations seen in

*Address correspondence to Philip J. Rosenthal, Department of Medicine, University of California, Box 0811, San Francisco, CA 94946. E-mail: philip.rosenthal@ucsf.edu other countries. The full clinical implications of these K13 mutations are as yet unclear, but recent trials in Rwanda ${ }^{9}$ and Uganda ${ }^{12}$ have shown excellent ACT treatment efficacy, perhaps due to continued excellent efficacy of artemisinin partner drugs. However, there is concern that Africa may see the pattern experienced in southeast Asia, with emergence of artemisinin resistance followed by decreased activity of partner drugs and then emergence of true ACT resistance. In fact, other data suggest that such resistance may already be emerging in Africa.

The WHO uses a cutoff of $90 \%$ to define acceptable malaria treatment efficacy. Use of a precise cutoff is somewhat problematic because measured efficacy may vary based on factors independent of drug resistance, including the immunity and genetics of local populations and details of trial design and data analysis, in particular varied molecular methods and analyses to distinguish recrudescences (true treatment failures) from new infections after therapy. ${ }^{13}$ Nonetheless, consideration of treatment efficacy over time is important.

Most studies of approved ACTs (artemether-lumefantrine, artesunate-amodiaquine, dihydroartemisinin-piperaquine, artesunate-mefloquine, and artesunate-pyronaridine) conducted in Africa in recent years following standard WHO protocols have shown excellent treatment efficacy. ${ }^{7}$ However, there have been important exceptions. A series of four studies conducted every 2 years in Angola from 2013 to 2019 showed treatment efficacies (values provided below were corrected by molecular methods to discriminate recrudescences from new infections, although analytical approaches varied) for artemether-lumefantrine below $90 \%$ for Zaire Province in 2013 and 2015 (but not 2017 or 2019) and for Lunda Sul Province in 2019; efficacies for the other tested ACTs (artesunateamodiaquine and dihydroartemisinin-piperaquine) were mostly $>95 \% .{ }^{14-17}$ A new report in this issue of the American Journal of Tropical Medicine and Hygiene describes the results of $A C T$ treatment trials conducted at four sites in the Democratic Republic of the Congo (DRC) in 2017-2018. ${ }^{18}$ Most efficacies were $>90 \%$, but in Mikalayi, which is near the border with Zaire Province, Angola, treatment efficacy was $86 \%$ for artemether-lumefantrine and $84 \%$ for dihydroartemisinin-piperaquine. Even more striking results were recently reported from Burkina Faso, with treatment efficacies of $74 \%$ and $76 \%$ for artemether-lumefantrine and $89 \%$ and $84 \%$ for dihydroartemisinin-piperaquine noted in two provinces. ${ }^{19}$ Importantly, the relatively low efficacy values in Angola and DRC were derived from a Bayesian algorithm for outcome assignment that can identify higher failure rates than the standard algorithm recommended by the $\mathrm{WHO},{ }^{20,21}$ and results from Burkina Faso are arguably difficult to interpret due to deviations from standard WHO protocols. ${ }^{22}$ Interestingly, for the reported studies from Angola, DRC, and Burkina Faso, sequencing did not identify K13 mutations associated with artemisinin resistance in southeast Asia. 
Considering the results described above, are we beginning to see ACT resistance in Africa? The answer is not yet clear, but two independent trends deserve careful attention. First, K13 mutations previously associated with artemisinin resistance have emerged in multiple African countries, albeit without apparent loss of ACT treatment efficacy. Second, ACT treatment efficacies below $90 \%$ have been seen in some other African countries without apparent emergence of relevant $\mathrm{K} 13$ mutations. How should we act on these findings? For the present, with limited evidence for increasing rates of ACT treatment failure, continued use of artemether-lumefantrine and other approved ACTs to treat malaria in Africa is appropriate. However, frequent surveillance across Africa is needed to identify further emergence of genetic polymorphisms that may mediate resistance to artemisinins and their partner drugs, ex vivo evidence of decreased drug susceptibility, or failures of ACT treatment efficacy in well-conducted clinical trials. For the future, emergence of ACT resistance may necessitate new therapeutic approaches for malaria in Africa.

Received July 13, 2021. Accepted for publication July 14, 2021.

Published online September 7, 2021.

Acknowledgments: I thank Pascal Ringwald, WHO, for critical review of this article. The author's research is funded by grants from the National Institutes of Health and Medicines for Malaria Venture.

This is an open-access article distributed under the terms of the Creative Commons Attribution (CC-BY) License, which permits unrestricted use, distribution, and reproduction in any medium, provided the original author and source are credited.

\section{REFERENCES}

1. World Health Organization, 2020. World Malaria Report. Geneva, Switzerland: WHO.

2. Gething PW et al., 2016. Mapping Plasmodium falciparum mortality in Africa between 1990 and 2015. N Engl J Med 375: 2435-2445.

3. Ashley EA et al., 2014. Spread of artemisinin resistance in Plasmodium falciparum malaria. N Engl J Med 371: 411-423.

4. Ariey $\mathrm{F}$ et al., 2014. A molecular marker of artemisinin-resistant Plasmodium falciparum malaria. Nature 505: 50-55.

5. Na-Bangchang K, Ruengweerayut R, Mahamad P, Ruengweerayut K, Chaijaroenkul W, 2010. Declining in efficacy of a three-day combination regimen of mefloquine-artesunate in a multi-drug resistance area along the Thai-Myanmar border. Malar J 9: 273.

6. Amaratunga $\mathrm{C}$ et al., 2016. Dihydroartemisinin-piperaquine resistance in Plasmodium falciparum malaria in Cambodia: a multisite prospective cohort study. Lancet Infect Dis 16: 357365.

7. Conrad MD, Rosenthal PJ, 2019. Antimalarial drug resistance in Africa: the calm before the storm? Lancet Infect Dis 19: e338e358.
8. World Health Organization, 2020. Report on Antimalarial Drug Efficacy, Resistance and Response: 10 Years of Surveillance (2010-2019). Geneva, Switzerland: WHO.

9. Uwimana A et al., 2020. Emergence and clonal expansion of in vitro artemisinin-resistant Plasmodium falciparum kelch13 R561H mutant parasites in Rwanda. Nat Med 26: 1602-1608.

10. Uwimana A et al., 2021. Association of Plasmodium falciparum kelch13 R561H genotypes with delayed parasite clearance in Rwanda: an open-label, single-arm, multicentre, therapeutic efficacy study. Lancet Infect Dis (Epub ahead of print).

11. Asua $V$ et al., 2021. Changing prevalence of potential mediators of aminoquinoline, antifolate, and artemisinin resistance across Uganda. J Infect Dis 223: 985-994.

12. Yeka A et al., 2019. Comparative efficacy of artemetherlumefantrine and dihydroartemisinin-piperaquine for the treatment of uncomplicated malaria in Ugandan children. $J$ Infect Dis 219: 1112-1120.

13. Plucinski MM, Hastings IM, Moriarty LF, Venkatesan M, Felger I, Halsey ES, 2021. Variation in calculating and reporting antimalarial efficacy against Plasmodium falciparum in subSaharan Africa: a systematic review of published reports. Am J Trop Med Hyg 104: 1820-1829.

14. Plucinski MM et al., 2015. Efficacy of artemether-lumefantrine and dihydroartemisinin-piperaquine for treatment of uncomplicated malaria in children in Zaire and Uige Provinces, Angola. Antimicrob Agents Chemother 59: 437-443.

15. Plucinski MM et al., 2017. Efficacy of artemether-lumefantrine, artesunate-amodiaquine, and dihydroartemisinin-piperaquine for treatment of uncomplicated Plasmodium falciparum malaria in Angola. Malar J 16: 62.

16. Davlantes E et al., 2018. Efficacy and safety of artemetherlumefantrine, artesunate-amodiaquine, and dihydroartemisinin-piperaquine for the treatment of uncomplicated Plasmodium falciparum malaria in three provinces in Angola, 2017. Malar J 17: 144.

17. Dimbu PR et al., 2021. Continued low efficacy of artemetherlumefantrine in Angola in 2019. Antimicrob Agents Chemother 65: e01949-20.

18. Moriarty LF et al., 2021. Therapeutic efficacy of artemisininbased combination therapies in Democratic Republic of the Congo and investigation of molecular markers of antimalarial resistance. Am J Trop Med Hyg 105: 1067-1075.

19. Gansané A et al., 2021. Anti-malarial efficacy and resistance monitoring of artemether-lumefantrine and dihydroartemisininpiperaquine shows inadequate efficacy in children in Burkina Faso, 2017-2018. Malar J 20: 48.

20. Plucinski MM, Morton L, Bushman M, Dimbu PR, Udhayakumar $\mathrm{V}, 2015$. Robust algorithm for systematic classification of malaria late treatment failures as recrudescence or reinfection using microsatellite genotyping. Antimicrob Agents Chemother 59: 6096-6100.

21. Rasmussen C, Ringwald P, 2021. Continued low efficacy of artemether-lumefantrine in Angola? Antimicrob Agents Chemother 65: e01949-20.

22. Rasmussen C, Ringwald P, 2021. Is there evidence of antimalarial multidrug resistance in Burkina Faso? Malar J 20: 320. 